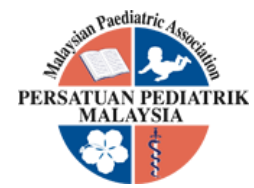

\title{
CONCEALED FOREIGN BODY ASPIRATION: A MASTER OF DISGUISE
}

\author{
Pravin Sugunan', Netia Jeganathan², Philip Rajan Devesahayam²,3
}

\begin{abstract}
Aspiration of a foreign body is rare in school-age children. This reports the 21-day journey of an 8-year-old girl who had a foreign body aspiration. She presented to our hospital after five days of respiratory distress. She subsequently required mechanical ventilation and was supported with triple inotropes. After 18 days, a foreign body was removed via rigid bronchoscopy, followed by a rapid recovery of the patient.
\end{abstract}

\section{Keywords:}

Foreign body; Paediatric; Aspiration

\section{Introduction}

Foreign body aspiration (FBA) is commonly seen in children less than 6 years of age. FBA in children usually presents with an initial episode of choking with subsequent respiratory symptoms. There may be cough, wheeze, or stridor, with decreased or abnormal breath sounds on examination. However, it can mimic other illnesses and cause difficulty in diagnosis. Unrecognized FBA can lead to serious, potentially life-threatening illness. Here we present an 8year-old child whose diagnosed as FBA with a which proved a challenged in diagnosis.

\section{Case Presentation}

SZ, an 8-year-5-month-old girl, presented to a government health clinic, with acute onset of breathlessness, gasping and a generalized tonic seizure in the evening. It was preceded by a fiveday history of progressively worsening cough and rhinorrhea. Her parents were poor historians (father had significant hearing and speech impairment, and mother had moderate intellectual impairment). History from her aunt revealed that there was no history of choking, witnessed foreign body aspiration or recent trauma. She was then referred to a District Specialist Hospital for further care.

Upon examination, the child was hypoxic with oxygen saturation of $71 \%$ in room air and symptoms of severe respiratory distress. Her chest was hyperinflated, with reduced breath sounds
Received: 11 February 2020; Accepted revised manuscript: 12 April 2020

Published online: 26 April 2020

bilaterally, and chest x-ray showed bilateral pneumonic changes. She required an emergency tracheal intubation, mechanical ventilation and fluid resuscitation. Following endotracheal intubation, SZ developed bilateral pneumothoraxes with subcutaneous emphysema over the neck and chest, which required placement of pleural drains bilaterally. She was initially treated at the District Specialist Hospital for suspected severe exacerbation of bronchial asthma secondary to bronchopneumonia. She was treated with nebulized and intravenous bronchodilators, nebulized ipratropium bromide, intravenous steroids, magnesium sulphate and antibiotics, but showed no improvement. Following stabilization, SZ was urgently transferred to out paediatric intensive care unit (PICU).

Upon arrival at a tertiary centre PICU, SZ was ventilated on moderate settings and required high inotropic support initially. A provisional diagnosis of severe bronchopneumonia with septicaemic shock was made, and she was also treated with broad spectrum antibiotics and oral Oseltamivir. In view of her extensive subcutaneous emphysema over the neck and upper chest and bilateral pneumothoraxes, there was a clinical suspicion of foreign body aspiration. However, parents repeatedly denied any history

${ }^{1}$ Department of Paediatrics, Hospital Raja Permaisuri Bainun, Perak, Malaysia

${ }^{2}$ Clinical Research Centre, Hospital Raja Permaisuri Bainun Perak, Malaysia

${ }^{3}$ Department of Otorhinolaryngology Head \& Neck Surgery, Hospital Raja Permaisuri Bainun PerakMalaysia Corresponding author: Netia Jeganathan, Clinical Research Centre, Hospital Raja Permaisuri Bainun, Jalan Raja Ashman Shah, 30990 Ipoh, Perak Tel: +605-2085000; Email: netia545@gmail.com 
suggestive of foreign body aspiration, and serial chest X-rays did not show any evidence of a foreign body. Her nasopharyngeal aspirate was positive for Parainfluenza 3 virus.

During the treatment to stabilize her, serial radiographs were performed and reported however none were suggestive of FBA. During the six days of treatment, SZ had persistent left lung collapse-consolidation and failed extubation twice, requiring prompt reintubation due to upper airway obstruction and distress. In view of recurrent extubation failure, a contrast enhanced computed tomography scan of the thorax (CECT thorax), was done and reported.

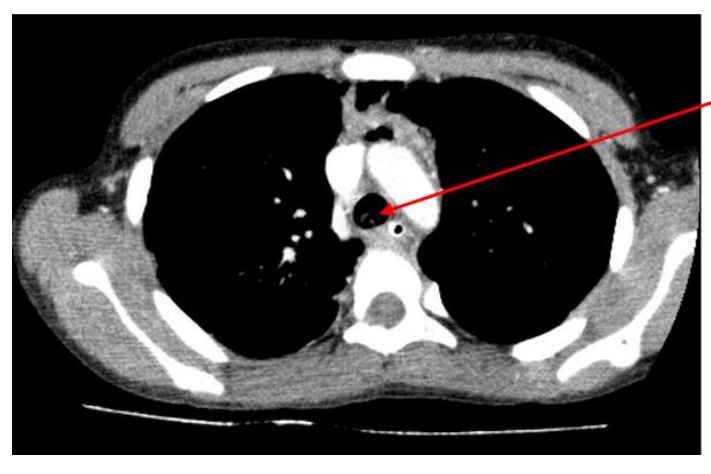

A.1) transverse view

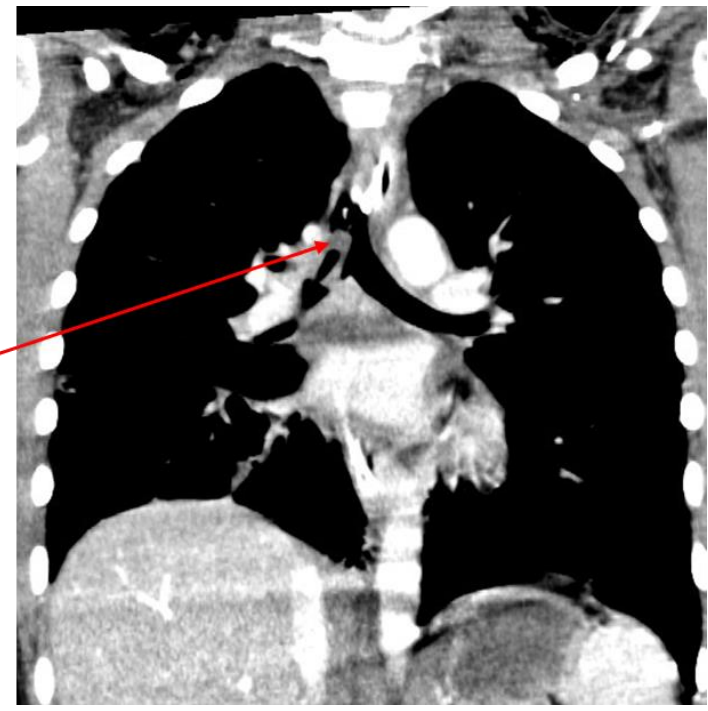

A. 2) vertical view

Figure A: CECT thorax in two views a) transverse and b) vertical, showing arrow (red) of suspicious FBA

CECT thorax reported a C-shaped well-defined structure (up to $60 \mathrm{HU}$ ) seen in the lower trachea, distal to and separated from the endotracheal tube. SZ was referred to the Otorhinolaryngology Head \& Neck Surgery department and on day 18, an urgent bronchoscopy was done. The foreign body was fished out via a rigid ventilating bronchoscope Karl Stroz $3.7 \mathrm{~mm}$ via the oral orifice.

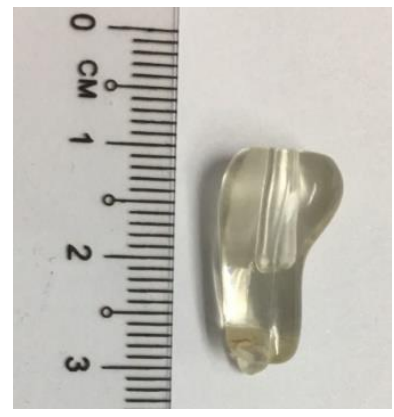

B. 1) Height $2.0 \mathrm{~cm}$

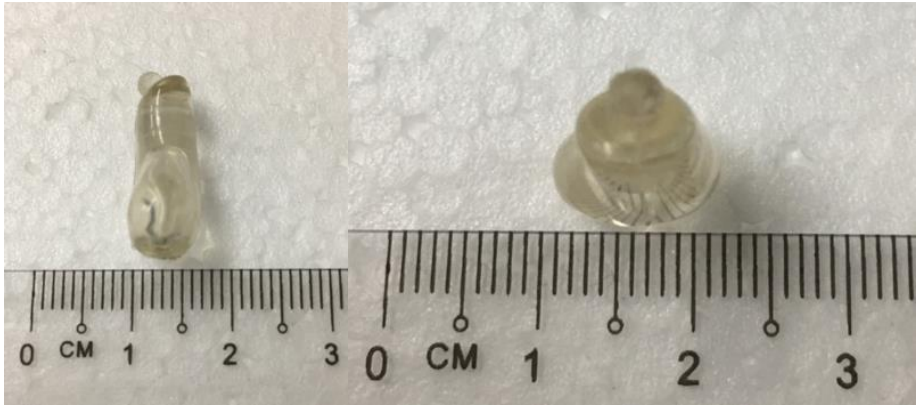

B. 2) Width $(0.5 \times 1.2) \mathrm{cm}$

Figure B: Concealed foreign body which was removed from SZ. 
SZ was successfully extubated following removal of the foreign body, showed steady improvement and was discharged home at day 21 . She went home with clear lungs and equal breath sounds. She was reviewed two weeks later in the paediatric clinic and was clinically well with complete resolution.

\section{Discussion}

Foreign body aspiration (FBA) occurs mainly in children under three years of age and is one of the most frequent causes of accidental death under 12 months of age, and continues to be a common problem in paediatrics that may have severe consequences, as it can result in both acute and chronic health problems [1].

FBA is a serious and potentially fatal condition. Early recognition and appropriate management are crucial to avoid complications and irreversible lung injury [2]. Our case illustrates the myriad difficulties associated with diagnosing foreign body aspiration in a school-age child, especially in the absence of a positive clinical history. The association between foreign body aspiration and air leak syndromes, such as pneumothoraxes, pneumomediastinum and subcutaneous emphysema, has been well described in the literature. The underlying pathophysiologic mechanism postulated is as follows: The aspirated foreign body leads to expiratory airflow obstruction, creating a ball-valve effect in the distal lung. The affected lung becomes hyperinflated, and a high pressure gradient is created between intra-alveolar air and the interstitial connective tissue. Subsequently, alveolar rupture occurs, with air tracking along fascial planes to the mediastinum, and thence to the subcutaneous tissues of the neck, chest, back, abdomen or face. Air leak can also extend into the pleural cavity, as in our patient $[3,4,5]$.

Chest radiographs are often utilized as initial tool of investigation for suspected foreign body aspiration. Radiological findings consistent with foreign body aspiration include atelectasis, pneumothorax, and air trapping. Radiopaque objects are readily diagnosed on chest radiograph. However, chest radiographs have been seen in multiple studies to be frequently normal, with one study citing a normal chest radiograph in $35 \%$ of cases of foreign body aspiration. This same study indicated that the most common abnormality found on radiograph was air trapping, present in 53\% of cases. The sensitivity and specificity of chest radiograph in diagnosing FBA has been documented as $67.9 \%$ and $71.4 \%$ respectively [6]. Therefore, a normal chest X-ray does not rule out FBA. Rather, direct identification via bronchoscopy is often needed.

In summary, FBA can mimic many other conditions, making it a challenging diagnosis. A high index of suspicion is needed to correctly diagnose FBA in children. Early bronchoscopy and foreign body removal can lead to an excellent clinical outcome.

\section{Conclusion}

This case report illustrates that foreign body aspiration in a school-age child may not be reliably excluded in the absence of a suggestive clinical history and typical chest x-ray findings. Foreign body aspiration may occur simultaneously with bronchopneumonia, making a timely diagnosis even more challenging. The presence of significant air leak, such as pneumothoraxes and subcutaneous emphysema, should alert clinicians to the possibility of an underlying concealed FBA.

If foreign body aspiration is suspected, the diagnostic and therapeutic modality of choice would be rigid bronchoscopy and removal of the foreign body. However, in a resource limited setting without on-site Paediatric Otorhinolaryngology or Paediatric Pulmonology services, a contrast-enhanced CT Thorax may be a pragmatic modality to facilitate detection of an aspirated foreign body, and help exclude other differential diagnoses, such as airway malacia or vascular rings.

\section{Ethical consideration}

The study team obtained written informed consent. All data collected were kept confidential, and no unique identifiers were collected.

\section{Funding}

This study was self-funded.

\section{Competing interests}

None.

\section{Provenance and peer review}

Not commissioned; externally peer reviewed.

\section{Data sharing statement}

All research data are securely archived at Clinical Research Centre, Hospital Permaisuri Bainun, Perak. The full anonymised dataset is available from the corresponding author. 


\section{Disclaimer}

The views, interpretations, implications, conclusions, and recommendations expressed in this case report are those of the authors alone and do not necessarily represent the opinions nor the views or policy of the Ministry of Health Malaysia.

\section{Acknowledgement:}

We would like to express our sincere gratitude to the following departments which have facilitated the production of this case report:

a. Department of Paediatrics, Raja Permaisuri Bainun Hospital.

b. Clinical Research Centre, Raja Permaisuri Bainun Hospital.

c. Department of Otorhinolaryngology Head and Neck Surgery, Raja Permaisuri Bainun Hospital.

Thank you for your guidance and cooperation which facilitated to the success of this research.

\section{References}

[1] Davina Stasia Teo, Zara Nasseri, Pravin Sugunan, Faizah Mohd Zaki, Tang Swee Fong, Bee-See Goh. Concealed foreign body aspiration presenting as subcutaneous emphysema. RMJ. 2016; 41(4): 499-501.

[2] Lluna J, Olabarri $M$, Domènech $A$ et all. Recommendations for the prevention of foreign body aspiration. An Pediatr (Barc) 2017 Jan;86(1): 50e1-50e6.

[3] Findlay CA, Morrissey S, Paton JY. Subcutaneous emphysema secondary to foreign-body aspiration. Pediatr Pulmonol 2003;36:81-2.

[4] Sodhi KS, Saxena AK, Singh M et al. New non invasive tool in pediatric patients with foreign body aspiration. Indian J Pediatr 2008;75:511-3.

[5] Aggarwal SK, Keshri A.Subcutaneous et al. Emphysema: An unusual presentation of an unsuspected foreign body bronchus in an adult patient. J Laryngol, Voice 2012;2:38-4.

[6] Caliskan E, Aliyev S, Habibi HA, et al. Utility of lung radiodensity ratios in diagnosis of radiolucent foreign body aspiration in children: a practical approach. Clinical Imaging. 2019 Mar - Apr; 54:178-182. 